\title{
Proporzwahrende Anpassung der Bundestagsgröße - ein Lösungsvorschlag für das Problem der negativen Stimmgewichte bei Bundestagswahlen
}

\author{
Daniel Lübbert, Felix Arndt und Friedrich Pukelsheim*
}

Das Bundesverfassungsgericht hat in seinem Urteil vom 3. Juli 2008 zum negativen Stimmgewicht im Bundeswahlgesetz dem Gesetzgeber aufgegeben, bis zum 30. Juni 2011 den monierten Defekt zu reparieren. ${ }^{1}$ Trotz der in spürbare Nähe gerückten Frist hat die Auseinandersetzung um das Wahlrecht bisher zu keiner Einigung auf ein Reformmodell geführt. In der jüngeren Diskussion ${ }^{2}$ wurden unter anderem einige fundamentale Alternativen zum gegenwärtigen Wahlsystem, von der Einführung eines reinen Mehrheitswahlrechts über ein Grabenwahlsystem ${ }^{3}$ bis hin zur Wahlorganisation mit Hilfe von Bundesstatt Landeslisten der Parteien, erwogen. Jeder dieser Vorschläge könnte einerseits den monierten Defekt im Wahlsystem abstellen, doch würden sie andererseits auch zu große Veränderungen entweder im Ergebnis (bei den Mehrheitsverhältnissen im Parlament) oder im Verfahren (bei der innerparteilichen Kandidatenaufstellung) nach sich ziehen, um der

* Die Autoren Daniel Lübbert und Felix Arndt sind Mitarbeiter der Wissenschaftlichen Dienste des Deutschen Bundestages. Der Beitrag gibt ausschließlich ihre persönliche Auffassung wieder.

1 Vgl. BVerfGE 121, S. 266 - 317. Zur Problematik und einigen Lösungsmöglichkeiten siehe Wolfgang Schreiber, BWahlG - Kommentar zum Bundeswahlgesetz, Köln 2009, S. 242 - 247; Dieter Nohlen, Erfolgswertgleichheit als fixe Idee oder: Zurück zu Weimar? Zum Urteil des Bundesverfassungsgerichts über das Bundeswahlgesetz vom 3. Juli 2008, in: ZParl, 40. Jg. (2009), H. 1, S. 179 - 195; Daniel Lübbert, Negative Stimmgewichte bei der Bundestagswahl 2009, in: ZParl, 41. Jg. (2010), H. 2, S. 278 - 289; Joachim Behnke, Überhangmandate und negatives Stimmgewicht: Zweimannwahlkreise und andere Lösungsvorschläge, in: ZParl, 41. Jg. (2010), H. 2, S. 247 - 260; Franz Urban Pappi / Michael Herrmann, Überhangmandate ohne negatives Stimmgewicht: Machbarkeit, Wirkungen, Beurteilung, in: ZParl, 41. Jg. (2010), H. 2, S. 260 278; Hans Meyer, Die Zukunft des Bundestagswahlrechts zwischen Unverständnis - obiter dicta, Interessenkalkül und Verfassungsverstoß, Baden-Baden 2010; Friedrich Pukelsheim / Matthias Rossi, Wahlsystemnahe Optionen zur Vermeidung negativer Stimmgewichte, in: JZ, 65. Jg. (2010), H. 5, S. 922 - 929; Olga Birkmeier / Kai-Friederike Oelbermann / Friedrich Pukelsheim / Matthias Rossi, Eine schonende Verbindung von Personen- und Verhältniswahl zum Abbau negativer Stimmgewichte bei Bundestagswahlen, KritV, 94. Jg. (2011), H. 1, S. 55 - 79; Gerd Strohmeier, Die Geister, die Karlsruhe rief - eine Replik auf die Beiträge zur Wahlsystemreform in Heft 2/2010 der ZParl, in: ZParl, 42. Jg. (2011), H. 1, S. 186 - 193.

2 Vgl. Joachim Behnke, Das Wahlsystem der Bundesrepublik Deutschland. Logik, Technik und Praxis der Verhältniswahl, Baden-Baden 2007; Florian Grotz, Verhältniswahl und Regierbarkeit: das deutsche Wahlsystem auf dem Prüfstand, in: Gerd Strohmeier (Hrsg.), Wahlsystemreform, ZPol-Sonderband, Baden-Baden 2009, S. 155 - 181; Daniel Lübbert, Negative Stimmgewichte und die Reform des Bundestagswahlrechts, Infobrief der Wissenschaftlichen Dienste des Deutschen Bundestages, Berlin 2009, http://www.bundestag.de/dokumente/analysen/2009/negative stimmgewichte.pdf (Abruf am 10. Juni 2011); Hans Meyer, Lösungsmöglichkeiten nach dem Wahlrechtsurteil des BVerfG vom 3. Juli 2008, in: DVBl, 124. Jg. (2009), H. 3, S. 137 - 146; Hans Meyer, a.a.O (Fn. 1).

3 Zur Kritik an Grabenwahlsystemen Ernst Gottfried Mahrenholz, Über den Satz vom zwingenden Grund, in: Gerd Pfeiffer / Udo Burgermeister / Gerald Roth (Hrsg.), Der verfaßte Rechtsstaat Festgabe für Karin Graßhof, Heidelberg 1998, S. 69 - 85, S. 81. 
notwendigen Mehrheit der Beteiligten akzeptabel zu erscheinen. Für die demokratische Legitimität des Wahlrechts wünschenswert wäre darüber hinaus eine Lösung, die nicht nur von der notwendigen, sondern von einer möglichst breiten Mehrheit im Parlament getragen wird.

Die Diskussion konzentriert sich daher zunehmend auf Reformmodelle, die vielfach als „minimal-invasive“ Fortentwicklungen des bestehenden und in seinen Grundzügen bewährten Systems einer mit der Personenwahl verbundenen Verhältniswahl bezeichnet wurden. Das Prädikat „minimal-invasiv“ wurde dabei durchweg uneinheitlich gebraucht und alternativ entweder auf das Verfahren oder auf das Ergebnis der Sitzzuteilung bezogen. ${ }^{4}$ Entsprechend groß ist die Anzahl der Reformmodelle, die dieser Gruppe zugeordnet werden können: „getrennte Stimmenauswertungen in den Ländern“ (Abschaffung der aggregierten Stimmenauswertung auf Bundesebene beziehungsweise der zweistufigen Sitzzuteilung), „Listenverbindungen abschaffen“ (sämtliche Landeslisten aller Parteien konkurrieren bei der Sitzzuteilung direkt auf Bundesebene miteinander), „Listenverbindungen bei Bedarf lösen" (ähnliches Prinzip, jedoch werden nur diejenigen Landeslisten sukzessive aus ihrer jeweiligen Listenverbindung gelöst, die bereits Überhangmandate oder zumindest keine Listenmandate erzielt haben), oder „Kompensation aus anderen Landeslisten“ (erzielt eine Partei in einigen Ländern Überhangmandate, so muss sie in anderen Ländern auf ebenso viele Listenmandate verzichten).

Auch diese Vorschläge wären durchweg geeignet, das problematische Phänomen der negativen Stimmgewichte entweder gar nicht mehr oder zumindest merklich seltener als bisher auftreten zu lassen. Manche Lösungen setzen allerdings nur an dem Entstehungsmechanismus für negative Stimmgewichte an, der im geltenden Wahlrecht die primäre Rolle spielt. Sie verhindern aber nicht andere Mechanismen, die zwar weniger wahrscheinlich zu negativen Stimmgewichten führen, diese aber immer noch in einer nicht nur durch unvermeidbare mathematische Rundungsphänomene verursachten Weise begünstigen. ${ }^{5} \mathrm{Ob}$ diese unterschiedlichen Entstehungsmechanismen trotzdem zu unterschiedlichen verfassungsrechtlichen Würdigungen führen, soll hier nicht vertieft erörtert werden. ${ }^{6}$

Im Laufe der Diskussion ist deutlich geworden, dass jedes der Reformmodelle neben Vorteilen auch spezifische Nachteile gegenüber dem bisherigen Wahlsystem aufweist. So stellt etwa das Kompensationsmodell zwar den Proporz zwischen den Parteien entsprechend ihres Zweitstimmenanteils wieder her, verstärkt im Gegenzug bei den Parteien mit Überhangmandaten aber die Verzerrung des föderalen Proporzes, die schon heute zwischen Landeslisten mit Überhängen und solchen ohne zu verzeichnen ist. Zudem kann dieses

4 Dies sollte nicht darüber hinwegtäuschen, dass einige der Vorschläge in demokratietheoretischer Hinsicht tiefgreifende Veränderungen mit sich brächten.

5 In diesen Fällen wandern zum Beispiel durch Stimmenverluste einer Landesliste frei werdende Mandate nicht automatisch zu einer anderen Landesliste der gleichen Partei, sondern kommen erst über den Umweg über die Oberverteilung mit einiger Wahrscheinlichkeit dort an.

6 Dem Umstand, dass eine der vom Bundesverfassungsgericht in einer Nebenbemerkung angeregten Lösungen (BVerfGE 121, S. 266 (S. 307, S. 315)) bei Lichte besehen nicht alle Entstehungsmechanismen ausschließen kann, sollte allerdings nicht überinterpretiert werden. Die Gründe der Entscheidung enthalten keine Anzeichen, dass dem BVerfG die alternativen Entstehungsmechanismen überhaupt vor Augen standen. Ein solches obiter dictum entbindet den Gesetzgeber daher nicht von seiner Pflicht zur sorgfältigen und umfassenden verfassungsrechtlichen Prüfung. 
Modell dazu führen, dass eine Partei in manchen Bundesländern gar nicht mehr durch Abgeordnete vertreten ist, obwohl sie dort einen ansehnlichen Anteil der Zweitstimmen erhalten hat.

Vor diesem Hintergrund soll hier eine Lösungsoption dargestellt werden, die wir für besonders systemnah halten. Der Ansatz beruht auf der Idee, die Beweglichkeit der Bundestagsgröße systemstabilisierend einzusetzen. ${ }^{7}$ Schon heute gilt, dass die in $\$ 1$ BWahlG genannte gesetzliche Bundestagsgröße von 598 Sitzen aufgrund von Überhangmandaten regelmäßig überschritten wird und de facto dadurch ihre Beweglichkeit beweist. Anstatt diesen Effekt nur passiv hinzunehmen, setzt das hier vorgestellte Anpassungsverfahren die Beweglichkeit der Bundestagsgröße aktiv zur Problemlösung ein. Ausgehend von einer Anfangsgröße wird die Zahl der Sitze möglichst sparsam so nach oben angepasst, dass die beiden im Bundeswahlgesetz vorgesehenen Komponenten der Personenwahl und der Verhältniswahl konfliktfrei miteinander verbunden werden.

Das Verfahren steht insoweit in erkennbarem Zusammenhang mit Vorschlägen, die bisher unter dem Begriff „Ausgleichsmandate“ diskutiert wurden. Allerdings wurde darunter bisher meist eine Lösung gefasst, die das Sitzzuteilungssystem ansonsten unverändert lässt und nur die Hausgröße so lange heraufsetzt, bis alle Überhangmandate proportionalisiert sind. Dies hat den Vorteil, sowohl den Parteienproporz als auch den föderalen Proporz perfekt wiederherzustellen, allerdings um den Preis eines kaum mehr akzeptablen Parlamentswachstums (um über 200 Sitze unter aktuellen Verhältnissen). Im Unterschied dazu bewirkt das im Folgenden vorzustellende Modell nur einen begrenzten Ausgleich in dem Sinne, dass zwar der Parteienproporz auf Bundesebene, nicht aber der föderale Proporz zwischen den einzelnen Landeslisten vollständig um die durch Überhangmandate ausgelösten Verzerrungen korrigiert wird - mit dem Nebeneffekt eines deutlich begrenzteren Anwachsens der Sitzzahl im Bundestag. ${ }^{8}$

Das Anpassungsverfahren kann ohne Schwierigkeiten sofort umgesetzt und unmittelbar bei der nächsten Wahl angewendet werden. Wenn die mit dem Verfahren einhergehende moderate Vergrößerung des Bundestages zurückgefahren werden soll, um die derzeitige Regelgröße von etwa 600 Sitzen auch zukünftig im Mittel einzuhalten, so kann der Anfangsschritt bei nachfolgenden Wahlen auf niedrigerem Niveau starten. Eine solche Entscheidung sollte spätestens zu Beginn der nächsten Wahlperiode fallen, damit der sich daraus ergebende Neuzuschnitt der Wahlkreise rechtzeitig vorgenommen werden kann.

\section{Verfahrensweise}

Das Anpassungsverfahren besteht aus drei Stufen, (1) dem Anfangsschritt, (2) der Zwischenanpassung und (3) der Endzuteilung.

7 In diesem Sinne schon früher Joachim Behnke, Von Überhangmandaten und Gesetzeslücken, in: APuZ, B 52/2003, S. 21 - 28; Friedrich Pukelsheim / Sebastian Maier, Parlamentsvergrößerung als Problemlösung für Überhangmandate, Pattsituationen und Mehrheitsklauseln, in: ZParl, 39. Jg. (2008), H. 2, $312-322$.

8 Wie hier die alternative Begründung der Richter Limbach, Graßhof, Sommer und Hassemer in BVerfGE 95, S. 335 (S. 403). 
(1) Im Anfangsschritt wird eine vorläufige Sitzverteilung nach den unveränderten Prinzipien des bisherigen Wahlrechts berechnet. Zum Beispiel standen bei der Wahl 2009 am Anfang die gesetzlichen 598 Sitze für die Verteilungsrechnung zur Verfügung. Davon entfielen bundesweit auf die CDU 173 Sitze, deren Unterzuteilungen an die Landeslisten zu 21 Überhangmandaten führten. Daraus wird für die Zwischenrechnung ein Bedarf von $173+$ 21 = 194 CDU-Sitzen vorgemerkt. Bei der CSU ergaben sich im Anfangsschritt 42 Sitze. Da die CSU 45 Direktmandate gewann, wird hier für die Zwischenanpassung ein Bedarf von 45 CSU-Sitzen vorgemerkt. Bei den anderen Parteien (SPD, Linke, Grüne, FDP) ergeben sich keine Anfangsüberhangmandate und somit auch keine zusätzlichen Bedingungen für die Zwischenanpassung.

(2) In der Zwischenanpassung wird die Bundestagsgröße schrittweise so lange um jeweils einen Sitz erhöht und (nur) die Oberzuteilung an die Parteien auf Bundesebene wiederholt durchgeführt, bis für alle Parteien die vorgemerkten Sitzkontingente befriedigt und vom Zweitstimmenanteil der Partei auf Bundesebene abgedeckt sind. Zum Beispiel erreicht bei der Wahl 2009 erstmals bei einer Bundestagsgröße von 666 Gesamtsitzen die CDU das für sie vorgemerkte Kontingent von 194 Sitzen. Im Windschatten dieser Erhöhung wird das Sitzkontingent für die CSU (45) automatisch erreicht und mit 46 Sitzen sogar übertroffen. Damit ist für 2009 die Endgröße des Bundestages festgestellt, nämlich 666 Sitze.

(3) In der Endzuteilung erhält bundesweit jede Partei genauso viele Mandate, wie die Verhältnisrechnung mit dem Divisorverfahren mit Standardrundung (Sainte-Laguë / Schepers) für sie ausgibt. Mit der ermittelten Endgröße werden die Sitze in der Oberzuteilung den Parteien also im genauen Verhältnis ihrer Zweitstimmenerfolge zugeteilt. Auf Bundesebene wird dem Prinzip der Erfolgswertgleichheit der Wählerstimmen uneingeschränkt Genüge getan. In der zweiten Stufe, den Unterzuteilungsrechnungen, werden dann pro Partei die ihr zustehenden Sitze an ihre Landeslisten weitergereicht. Dabei kommt die direktmandatsbedingte Variante des Divisorverfahrens mit Standardrundung (Augsburger Zuteilungsverfahren) bei der Unterzuteilung zum Einsatz. Aufgrund des Einsatzes dieses Verfahrens und der zuvor durchgeführten Zwischenanpassung ist damit sichergestellt, dass für jede Landesliste mindestens so viele Sitze verfügbar sind, wie sie Direktmandatsgewinne vorweist, ohne dass die Gesamtsitzzahl einer Partei deswegen noch den Parteienproporz auf Bundesebene verletzen könnte.

Im Ergebnis stellt sich für alle Parteien ohne Überhangmandate eine Sitzverteilung zwischen ihren Landeslisten ein, die vollkommen analog ihrer Verteilung im bisherigen System ist (wenn auch auf etwas höherem Sitzzahlniveau). Für die Partei mit dem die Endgröße bestimmenden Anteil an Überhangmandaten hingegen bleibt die Unterzuteilung auch in der Endrechnung genau dieselbe, die sich bereits im Rahmen des Anfangsschritts eingestellt hatte. Am Beispiel der Bundestagswahl 2009 wäre demnach die CDU im Ergebnis des Anpassungsverfahrens mit denselben Direkt- und Listenmandatszahlen und durch dieselben Personen vertreten, wie dies im aktuellen Bundestag der Fall ist.

In Anlehnung an manche Landeswahlgesetze könnte man versucht sein, beim Anpassungsverfahren von regulären Mandaten auszugehen, die zusammen mit Überhangmandaten und Ausgleichsmandaten zur Endgröße führen. Wir halten einen solchen Sprachgebrauch für unglücklich, da es eine dreifache Klasseneinteilung von Bundestagsmandaten nicht gibt und nicht geben kann, weil das Grundgesetz allen Abgeordneten Statusgleichheit garantiert. Ausschlaggebend ist beim Anpassungsverfahren schließlich nur die Endgröße des Bundestags. Da diese gerade so festgelegt wird, dass alle Direktmandatsgewinne in die 
Verteilungsrechnung eingebettet werden können, bleiben im Endergebnis eben keine Mandate bestehen, die den Charakter von Überhangmandaten haben. ${ }^{9}$

Abbildung 1 gibt einen Überblick, wie viele zusätzliche Anpassungsmandate bei den letzten fünf Wahlen geschaffen worden wären. Dafür wurden alle fünf Wahlen mit dem Divisorverfahren mit Standardrundung (Sainte-Laguë / Schepers) ausgewertet, um Vergleichbarkeit zu erzielen. ${ }^{10}$ Wie man Abbildung 1 entnehmen kann, wäre es zu jeweils 34, 28, 9, 24 und 68 Anpassungsmandaten gekommen. Bei allen fünf Wahlen wäre die Endgröße durch die Partei bestimmt worden, die den größten Anfangsüberhang aufweist. Wird das Anfangsniveau herabgesetzt, sinkt auch die endgültige Bundestagsgröße. Bei einer Umrechnung der Wahlergebnisse von 2009 auf 270 Wahlkreise und damit einhergehenden 540 Ausgangssitzen wäre eine Endgröße von kaum mehr als 600 Sitzen herausgekommen. Wenn bei der nächsten Wahl Überhangmandate in ähnlich massiver Weise wie 2009 auftreten, wäre eine solche Absenkung des Anfangsniveaus zu erwägen. Wenn wie in früheren Jahren die Zahl der Anfangsüberhangmandate weitaus geringer ist, würde ein milderer Eingriff wohl ausreichen.

\begin{tabular}{|c|c|c|c|c|c|c|}
\hline \multicolumn{7}{|c|}{ Abbildung 1: Anfangs-und Endgrößen des Bundestages beim Anpassungsverfahren } \\
\hline Wahljahr & $\mathrm{A}$ & $\ddot{U}$ & Aussc & ggebende Sitzvormerkung & $\mathrm{Z}$ & $\mathrm{E}$ \\
\hline 1994 & 656 & 16 & $\mathrm{CDU}$ & $232+13=245$ & 34 & 690 \\
\hline 1998 & 656 & 13 & SPD: & $285+13=298$ & 28 & 684 \\
\hline $2002^{*}$ & 596 & 5 & SPD: & $247+4=251$ & 9 & 605 \\
\hline 2005 & 598 & 16 & SPD: & $213+9=222$ & 24 & 622 \\
\hline 2009 & 598 & 24 & $\mathrm{CDU}$ & $173+21=194$ & 68 & 666 \\
\hline \multicolumn{7}{|c|}{$\begin{array}{l}\text { Lesebeispiel: Bei der Wahl } 1994 \text { wären bei } A=656 \text { Anfangssitzen insgesamt } \ddot{U}=16 \text { Überhangmandate } \\
\text { angefallen. Für die CDU wären bei } 232 \text { Verhältnissitzen } 13 \text { Wahlkreissitze ungedeckt geblieben, so dass } \\
\text { ein Bedarf von } 245 \text { Sitzen entstanden wäre. Um das CDU-Kontingent entsprechend anzuheben, wäre } \\
\text { die Bundestagsgröße um Z = } 34 \text { Zusatzsitze (inklusive der } 16 \text { Überhangmandate) auf E = } 690 \text { Endsitze } \\
\text { anzupassen gewesen. } \\
\text { * Ohne Berücksichtigung der zwei PDS-Direktmandate. } \\
\text { Quelle: Eigene Berechnungen. }\end{array}$} \\
\hline
\end{tabular}

\section{Einordnung}

Das Anpassungsverfahren übernimmt zahlreiche Aspekte des aktuellen Wahlsystems und erhält so dessen bewährte Vorteile. Insbesondere bleibt das Prinzip der mit der Personenwahl verbundenen Verhältniswahl unverändert, also das System aus Erst- und Zweitstimme sowie die Zusammensetzung des Parlaments aus Direkt- und Listenmandaten.

9 Somit wird auch das Nachrückerurteil des Bundesverfassungsgerichts obsolet (siehe BVerfGE 97 (1998), S. 317 - 331). Um die auf Bundesebene garantierte Verhältnismäßigkeit dauerhaft einzuhalten, sollte ein Nachrücken aus einer Landesliste ermöglicht werden. Naheliegend wäre ein Nachrücken aus der Liste in das Bundesland, in dem ein Sitz frei wird. Alternativ möglich wäre aber auch das Nachrücken aus derjenigen Landesliste, die bei einem Absenken der Direktmandatsmindestbedingung als nächste am Zuge wäre.

10 Für die Wahlen 1994 bis 2005 war die gesetzlich vorgeschriebene Zuteilungsmethode das HareQuotenverfahren mit Ausgleich nach größten Resten. 
In der Anfangsrechnung ist das Verhältnis von Direkt- zu Listenmandaten wie bisher genau hälftig. Ergeben sich in dieser anfänglichen Rechnung keine Überhangmandate, besteht kein Anlass für weitere Eingriffe, und das Verhältnis bleibt genau hälftig. Andernfalls werden Anpassungsmandate geschaffen, wodurch die Zahl der Mandate anwächst - wie dies bereits heute aufgrund von Überhangmandaten der Fall sein kann. Dieses Wachstum ist nicht fest vorgegeben, sondern passt sich dynamisch an die jeweiligen Notwendigkeiten des Wahlergebnisses an, um alle sich anfänglich abzeichnenden Überhangmandate schließlich noch in die Verhältnisrechnung einzubetten.

Die Einteilung in Wahlkreise und die Vertretung jedes einzelnen Wahlkreises durch den siegreichen Kandidaten bleiben ebenfalls unangetastet. Wie gewohnt bildet sich auch die föderale Struktur im Wahlsystem ab. Die Aufstellung der Landeslisten verbleibt in der Hoheit der Landesverbände der Parteien.

Zusammen mit der bisherigen Stimmgebung bleibt auch die aggregierte Auswertung der Zweitstimmenergebnisse auf Bundesebene bestehen. Wie bisher werden aus den Stimmenzahlen vermittels Oberzuteilung und den jeweiligen Unterzuteilungen die Sitzzahlen berechnet. Das Anpassungsverfahren dient einzig dazu, ausgehend von einer vom Gesetzgeber vorgegebenen Anfangsgröße die Endgröße des Bundestages so zu gestalten, dass die verbundene Auswertung von Erst- und Zweitstimme problemlos möglich wird.

Beim Anpassungsverfahren ist die Parlamentsgröße variabel, um sie auf das jeweilige Wahlergebnis abzustimmen. Diese Variabilität stellt keine grundsätzliche Neuerung dar, denn durch Überhangmandate konnte sich schon in der Vergangenheit die Bundestagsgröße nach oben verändern. Dennoch setzt das Anpassungsverfahren die Bereitschaft voraus, die Vorab-Kontrolle des Gesetzgebers über die Parlamentsgröße noch etwas weiter abzugeben als bisher. Auch ohne die Möglichkeit der Detailsteuerung hat der Bundestag jedoch weiterhin die Option, der zukünftigen Entwicklung seiner Größe eine Tendenz und eindeutige Richtung aufzuprägen, indem er beispielsweise die Anzahl der Wahlkreise reduziert.

\section{Eigenschaften}

Die Stärkeverhältnisse zwischen den Parteien auf Bundesebene (Parteienproporz) werden trotz anfänglicher Unausgewogenheiten durch Überhänge im Zuge des Anpassungsverfahrens makellos wiederhergestellt. Es verbleiben allenfalls unvermeidliche Rundungstoleranzen. Der auf den Stimmzetteln eingedruckte Hinweis, dass die Zweitstimme maßgeblich ist für die Stärkeverhältnisse der Parteien, erhält so eine noch stärkere Gültigkeit.

Für Parteien ohne Anfangsüberhangmandate werden die Stärkeverhältnisse zwischen den verschiedenen Landeslisten wie bisher vollständig abgebildet. Dieser föderale Proporz wird sogar auch für solche Parteien vollkommen wiederhergestellt, die zwar anfänglich Überhangmandate vorweisen, die dann aber im Rahmen der Anpassung der Sitzzahl proportionalisiert werden. Allerdings wird eine Partei, deren anfängliche Überhänge den Ausschlag geben, die Verzerrung des parteiinternen föderalen Proporzes nicht korrigiert, weil deren Direktmandatsgewinne in der Endrechnung nicht angetastet werden (die Unterzuteilung innerhalb dieser Partei wird zwischen Anfangs- und Endzuteilung nicht verändert). Aber die Verzerrung wird auch nicht weiter verstärkt, wie dies etwa beim Verfahren der Kompensation aus anderen Landeslisten der Fall wäre. Vielmehr bleibt es für die Partei mit den ausschlaggebenden Anfangsüberhangmandaten auch im Endergebnis bei eben der fö- 
deralen Verzerrung, die sich in der Anfangsrechnung eingestellt hat und die sich genauso auch im geltenden Wahlsystem ergeben hätte.

Die Repräsentation der einzelnen Bundesländer im Bundestag, summiert über alle Parteien, wird vom Anpassungsverfahren daher in geringerem Ausmaß berührt als im geltenden Wahlsystem. Die zusätzlich geschaffenen Anpassungssitze kommen nämlich im Verhältnis der Zweitstimmen auch den nicht mit Überhangmandaten versehenen Landesverbänden der anderen Parteien zu Gute. Dadurch wirken sie der Verzerrung der proportionalen Repräsentation der Bundesländer entgegen. ${ }^{11}$

Das vom Bundesverfassungsgericht monierte negative Stimmgewicht wird durch das Anpassungsverfahren weitgehend unmöglich gemacht. Dies ist wohl am leichtesten nachzuvollziehen, wenn man sich die konkrete Situation 2005 mit der Nachwahl im Wahlkreis Dresden I vor Augen hält. Seinerzeit erhielt die CDU 173 Verhältnissitze; zuzüglich der sieben Überhangmandate hatte sie 180 Sitze. Hätten in Dresden I zehntausend CDU-Anhänger mehr ihre Zweitstimme der CDU gegeben, so wäre eines der sächsischen CDUÜberhangmandate proportionalisiert worden. Bundesweit hätte die CDU so nur 173 Verhältnissitze plus sechs Überhangmandate, also 179 Sitze, bekommen und wäre im Verhältnis zu den anderen Mitbewerbern geschwächt worden. Dies ist der beanstandete Effekt des negativen Stimmgewichts.

Das Anpassungsverfahren hätte nun im ersten Fall die Bundestagsgröße auf 621 angepasst, um die 180 CDU-Sitze verhältnismäßig zu rechtfertigen. Im zweiten Fall hätte es die Parlamentsgröße auf 617 angepasst, um die 179 CDU-Sitze ins rechte Verhältnis setzen zu können. Zehntausend CDU-Zweitstimmen mehr hätten also dazu geführt, dass die makellose Verhältnismäßigkeit nicht erst bei 621, sondern schon auf dem Niveau von 617 Endsitzen hergestellt worden wäre. Statt eines negativen Stimmgewichts bleibt also allenfalls der eigenartige Effekt übrig, dass mehr Wähleraktivierung zu einem kleineren Bundestag führen kann. Verfassungsrechtlich dürfte es jedoch unerheblich sein, ob Erfolgswertgleichheit der Wählerstimmen auf dem Niveau von 621 oder von 617 Sitzen hergestellt wird.

Tatsächlich hätten 2005 nicht die sieben Überhangmandate der CDU die endgültige Bundestagsgröße bestimmt, sondern die neun Überhangmandate der SPD. Zusammen mit den 213 Verhältnissitzen hätte die SPD 222 Sitze erhalten. Um diese ins rechte Verhältnis zu setzen, hätte das Anpassungsverfahren zu einer endgültigen Bundestagsgröße von 622 Sitzen geführt. Der eben beschriebene Nebeneffekt wäre also gar nicht sichtbar geworden, weil nicht das CDU-, sondern das SPD-Kontingent das Niveau für die Endzuteilung festgelegt hätte.

\section{Verfahrensvarianten}

Als Zwischenfazit kann festgehalten werden, dass das oben vorgestellte Anpassungsverfahren alle wesentlichen Vorzüge des bewährten Bundestagswahlsystems erhält, das Problem der negativen Stimmgewichte aus praktischer Sicht löst und als wesentlichen Nachteil nur eine moderate Parlamentsvergrößerung nach sich zieht. Diese fällt wesentlich geringer aus als in den bisher diskutierten Modellen mit Ausgleichsmandaten, die zu etwa 800 Sitzen

11 Vgl. auch BVerfG 95, S. 335 (S. 403, alternative Begründung). 
führen würden, und ergibt für den aktuellen Bundestag eine Endgröße nach Anpassung von 666 Sitzen. Diese Größe liegt durchaus im Rahmen dessen, was in den 1990er Jahren üblich war (vgl. Abbildung 1).

Erscheint die Zahl dennoch zu hoch, existieren als Alternative oder Ergänzung zum oben diskutierten „einfachen“ Anpassungsverfahren zusätzlich Optionen, um die Endgröße tendenziell weiter abzusenken. Der schlichteste Ansatz hierzu liegt in einer Deckelung der Anpassung. Begrenzte man die zusätzlichen Anpassungsmandate im Vergleich zu den ursprünglichen Überhangmandaten etwa auf das Verhältnis 1:1, so würde dies bedeuten, dass zu 24 Überhangmandaten höchstens 24 Ausgleichsmandate hinzukommen dürfen. Der aktuelle Bundestag hätte dann mit insgesamt 48 Zusatzmandaten eine Größe von 646. Ein solches 1:1-Verhältnis wäre in dem Sinne ein scharfer Deckel, als er bei vergangenen Bundestagswahlen schon mehrfach konkret zur Anwendung gekommen wäre und den Ausgleich tatsächlich begrenzt hätte. Als Alternative zu dieser Art von festem Verhältnis könnte auch eine absolute Maximalgröße des Bundestages (zum Beispiel 660 Sitze) fest vorgegeben werden; möglicherweise ließe sich diese Art von Begrenzung schlüssiger mit der Arbeitsfähigkeit des Parlaments begründen. ${ }^{12}$

Problematisch an einem scharfen Deckel wäre für den Fall der Wahl 2009 zumindest, dass eine nennenswerte Zahl nicht ausgeglichener Überhangmandate verblieben wäre, die negative Stimmgewichte hätte zur Folge haben können. Problematisch könnte darüber hinaus sein, dass das Landesverfassungsgericht Schleswig-Holstein im Sommer 2010 eine gedeckelte Ausgleichsregelung im Landtagswahlrecht - die als Nebeneffekt im konkreten Fall die Mehrheitsverhältnisse im Landtag umdrehte - für verfassungsrechtlich unzulässig erklärt hat. ${ }^{13}$ Auch wenn dieses Urteil aufgrund unterschiedlicher verfassungsrechtlicher Vorgaben in der Landesverfassung und im Grundgesetz nicht unmittelbar auf das Bundestagswahlrecht übertragbar ist, wirft es doch einen Schatten auf die Diskussion um die enge Deckelung eines Ausgleichs im Bundestagswahlrecht.

Weniger problematisch mag die Lage zu bewerten sein, wenn eine Deckelung großzügiger gestaltet wird. In der Gesamtschau aller vergangenen Bundestagswahlen wäre bisher maximal ein Verhältnis von 1,83:1 nötig gewesen, um im Rahmen des Anpassungsverfahrens den Parteienproporz bei anfänglichen Überhangmandaten wiederherzustellen. Eine Deckelung des Verhältnisses Ausgleichsmandate : Überhangmandate etwa bei 2:1 hätte sich daher historisch noch nie als praktisch begrenzender Faktor erwiesen und daher auch unmöglich die Regierungsmehrheiten verändern können. Eine solche großzügige Deckelung hätte dennoch den positiven Effekt, in zukünftigen Fällen von „exotischeren“ Wahlergebnissen ein möglicherweise unangemessen großes Anwachsen des Parlaments zuverlässig zu verhindern. Ein solches Szenario wäre die Vorstellung, bei der Wahl 2009 hätte die CDU bundesweit kein einziges, die CSU in Bayern aber - wie geschehen - drei Überhangmandate errungen. Wegen des kleinen Zweitstimmenanteils der CSU auf Bundesebene hat diese

12 Vgl. zur Zulässigkeit einer Begrenzung einer Anpassung unter dem Gesichtspunkts der Arbeitsund Funktionsfähigkeit des Parlaments BVerfGE 95, S. 335 (S. 404).

13 Vgl. Hans Meyer, Schleswig-Holsteinisches Landeswahlgesetz unter Normenkontrolle und Wahlprüfung: Wahlgewitter mit gebremstem Blitz, in: NordÖR, 13. Jg. (2010), H. 11, S. 421 - 429; Heiko Holste, Neuwahl-Urteil in Schleswig-Holstein: Menetekel für die Reform des Bundestagswahlrechts, in: Recht und Politik: Vierteljahreshefte für Rechts- und Verwaltungspolitik, 46. Jg. (2010), H. 4, S. 193 - 199. 
Partei im Ausgleichsverfahren eine große „Hebelwirkung“"14: Für die drei CSU-Überhangmandate wären allein 40 Zusatzmandate für die anderen Parteien erforderlich - ein Verhältnis, das kaum mehr als angemessen bezeichnet werden kann. Mit einer Deckelung im Verhältnis 2:1 hingegen hätten drei CSU-Überhänge allenfalls zu einem Bundestag mit 607 Sitzen geführt. Trotz verbleibender, nicht vollkommen ausgeglichener Überhänge wäre das Problem der negativen Stimmgewichte aus praktischer Sicht gelöst, da die externen Überhangmandate der CSU ohnehin nicht zu negativem Stimmgewicht führen können.

Um zu verhindern, dass - wie in Schleswig-Holstein geschehen - die Deckelung zu einer Umkehrung knapper Mehrheitsverhältnisse im Parlament führt, kann zusätzlich vorgesehen werden, dass der Deckel bei Bedarf sukzessive um je einen Sitz gelockert wird, bis die Mehrheitsverhältnisse bei den Stimmenergebnissen sich auch in Mehrheiten nach Abgeordnetenzahlen widerspiegeln. So bliebe der Wählerwille unter allen Umständen auch im Parlament gewahrt. ${ }^{15}$

Dennoch bleibt es legitim, sich dem Gedanken einer Deckelung grundsätzlich zu verschließen. In diesem Fall könnten andere Strategien in Frage kommen, um die Endgröße beim Anpassungsverfahren weiter zu reduzieren. ${ }^{16}$ Denkbar wäre zum Beispiel die Kombination des oben vorgestellten Anpassungsverfahrens mit dem Modell einer Kompensation von Überhangmandaten aus anderen Landeslisten, das der Anpassung vorgeschaltet würde. ${ }^{17}$ Das wesentliche Problem dieser Lösung ist bekanntlich die verstärkte Verzerrung des innerparteilichen föderalen Proporzes. So hätten bei der Wahl 2009 mehrere CDU-Landesverbände auf alle ihre Listenmandate verzichten müssen, um die Überhänge der anderen CDU-Landesverbände vollständig zu kompensieren. Begrenzte man diesen Effekt zum Beispiel durch die Regelung, dass kein Landesverband mehr als die Hälfte der ihm eigentlich zustehenden Listenmandate zu innerparteilichen Kompensationszwecken abgeben muss, wären der CDU bei der Wahl 2009 statt 21 nur noch 13 Überhangmandate verblieben. Diese - und auch die drei CSU-Überhangmandate - hätten im anschließenden Anpassungsverfahren durch 27 Zusatzmandate für die anderen Parteien ausgeglichen werden können. Dank der vorgeschalteten Kompensationslösung hätte so die Endgröße des aktuellen Bundestages bei 641 statt 666 Sitzen gelegen.

\section{Systemkontinuität}

Zusammenfassend stellt das oben vorgestellte Anpassungsverfahren die gewünschte Verhältnismäßigkeit auf der Ebene der Oberzuteilung ohne Abstriche her und sichert dadurch die

14 Vgl. Hans Meyer, a.a.O (Fn. 1)

15 Die Mehrheitstreue als Anforderung für ein demokratisches Wahlsystem hebt das BVerfG in seinem Urteil zum Vertrag von Lissabon hervor, BVerfGE 123, S. 267 (S. 342 f., S. 372 f.). Angedeutet auch in BVerfGE 121, S. 266 (S. 305).

16 Neben der oben aufgeführten sind weitere Kombinationen erwägenswert, so zum Beispiel auch die Kopplung eines gedeckelten Anpassungsverfahrens mit einer nachgeschalteten Lösung von Listenverbindungen im Falle von Überhangmandaten. Nicht empfehlenswert scheint hingegen die Verbindung des Anpassungsverfahrens mit dem Prinzip „Länder als getrennte Wahlgebiete“ (länderweiser Ausgleich), da diese Kombination die spezifischen Nachteile beider Ansätze verstärkt.

17 In diese Richtung gingen bereits die Überlegungen der alternativen Begründung in BVerfGE 95, S. 335 (S. 404). 
Erfolgswertgleichheit der Wählerstimmen. Negative Stimmgewichte können in aller Regel nicht mehr auftreten. Viele weitere Vorteile des bisherigen Bundestagswahlsystems bleiben unverändert erhalten. Im Gegenzug setzt das Modell einzig die Bereitschaft voraus, die gesetzgeberische Kontrolle über die Parlamentsgröße ein Stück weiter abzugeben, als dies wegen der Möglichkeit von Überhängen bisher schon unvermeidlich ist.

Angewandt auf die Wahlergebnisse 2009 führt das Anpassungsverfahren zu einer Veränderung der Gesamtsitzzahl von 622 auf 666. Wird dies - trotz Vergleichbarkeit mit den in den 1990er Jahren üblichen Bundestagsgrößen - als zu viel empfunden, so verbleiben mindestens drei Alternativen, um die Endgröße im Zuge des Anpassungsverfahrens weiter abzusenken: Erstens wäre es möglich, die Anpassung maßvoll zu deckeln. Zweitens könnte dem Anpassungsverfahren eine Teil-Kompensation von Überhangmandaten aus anderen Landeslisten vorgeschaltet werden. Unabhängig davon könnte drittens die Anzahl der Wahlkreise von derzeit 299 auf zum Beispiel 275 reduziert werden, um die Endgröße des Bundestages in die Nähe der im gegenwärtigen Bundeswahlgesetz genannten Regelgröße (598) zurückzuführen. 